\title{
Microbiome patterns across the gastrointestinal tract of the rabbitfish Siganus fuscescens
}

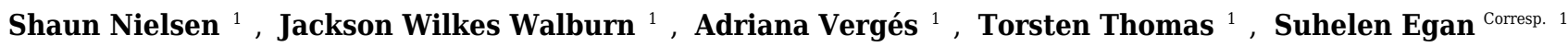 \\ ${ }^{1}$ Centre for Marine Bio-Innovation and School of Biological Earth and Environmental Sciences, University of New South Wales, Sydney, Australia \\ Corresponding Author: Suhelen Egan \\ Email address: s.egan@unsw.edu.au
}

Most of our knowledge regarding the biodiversity of gut microbes comes from terrestrial organisms or marine species of economic value, with less emphasis on ecologically important species. Here we investigate the bacterial composition associated with the gut of Siganus fuscescens, a rabbitfish that plays an important ecological role in coastal ecosystems by consuming seaweeds. Members of the Firmicutes, Bacteroidetes and deltaProteobacteria were among the dominant taxa across samples taken from the contents and the walls (sites) of the midgut and hindgut (location). Despite the high variability among individual fish, we observed statistically significant differences in beta-diversity between gut sites and gut locations. Some bacterial taxa low in abundance in the midgut content (e.g. Desulfovibrio) were found in greater abundances on the midgut wall and within the hindgut, suggesting that the gut may select for specific groups of environmental and/or food-associated microorganisms. In contrast, some distinct taxa present in the midgut content (e.g. Synechococcus) were noticeably reduced in the midgut wall and hindgut, and are thus likely to be representative of transient microbiota. This is the first assessment of the bacterial diversity associated with the gut of $S$. fuscescens and highlights the need to consider the variability across different gut locations and sites when analyzing fish gut microbiomes. 
1

2 Title: Microbiome patterns across the gastrointestinal tract of the rabbitfish Siganus fuscescens

3

4 Shaun Nielsen, Jackson Wilkes Walburn, Adriana Vergés, Torsten Thomas, Suhelen Egan*

5

6 Centre for Marine Bio-Innovation and the School of Biological Earth and Environmental

7 Science, University of New South Wales, Australia

8

$9 *$ Corresponding Author

10 Centre for Marine Bio-Innovation, School of Biological Earth and Environmental Science, 11 University of New South Wales, Australia

12 Email: s.egan@unsw.edu.au

13 Phone: + $61(0) 293858569$

14

15 


\section{Abstract}

17 Most of our knowledge regarding the biodiversity of gut microbes comes from terrestrial 18 organisms or marine species of economic value, with less emphasis on ecologically important 19 species. Here we investigate the bacterial composition associated with the gut of Siganus 20 fuscescens, a rabbitfish that plays an important ecological role in coastal ecosystems by 21 consuming seaweeds. Members of the Firmicutes, Bacteroidetes and delta-Proteobacteria were 22 among the dominant taxa across samples taken from the contents and the walls (sites) of the 23 midgut and hindgut (location). Despite the high variability among individual fish, we observed 24 statistically significant differences in beta-diversity between gut sites and gut locations. Some 25 bacterial taxa low in abundance in the midgut content (e.g. Desulfovibrio) were found in greater 26 abundances on the midgut wall and within the hindgut, suggesting that the gut may select for 27 specific groups of environmental and/or food-associated microorganisms. In contrast, some 28 distinct taxa present in the midgut content (e.g. Synechococcus) were noticeably reduced in the 29 midgut wall and hindgut, and are thus likely to be representative of transient microbiota. This is 30 the first assessment of the bacterial diversity associated with the gut of $S$. fuscescens and 31 highlights the need to consider the variability across different gut locations and sites when 32 analyzing fish gut microbiomes. 


\section{Introduction}

35

36 Microbial symbioses are key to the survival of multicellular organisms with individuals capable 37 of harboring diverse communities of beneficial microorganisms. In particular, gut bacteria play a 38 vital role not only in host nutrition, but also in mediating host immune functions, host 39 development and even influencing host behavior (Collins \& Bercik, 2009, Hansen et al., 2012, 40 Greenhalgh et al., 2016). Recent studies have highlighted the diversity and role of gut 41 microbiomes in terrestrial animals, with a particular focus on humans (see reviews by (Hacquard 42 et al., 2015, Colston \& Jackson, 2016, Greenhalgh et al., 2016)). In contrast, relatively little is 43 known about the gut microbiota of marine vertebrates, with most studies focusing on species of 44 economic value (Colston \& Jackson, 2016).

45

46 Fish are the most diverse group of vertebrates in the marine environment. They occupy a wide 47 range of habitats, have highly varying diets and often play important trophic and ecological roles. 48 While our understanding of the role and characteristics of fish gut microbiota is still very limited, 49 studies to date indicate that the bacterial composition of the fish gastrointestinal (GI) track is species-specific and influenced by host physiology as well as environmental conditions, such as 51 diet, salinity and geographic location (Sullam et al., 2012, Wong \& Rawls, 2012, Clements et al., 52 2014, Romero et al., 2014, Givens et al., 2015).

53

54 Diet is arguably one of the strongest influences on gut microbiotia and studies in mammals 55 suggest a trend of increasing bacterial diversity from carnivores to omnivores to herbivores (Ley et al., 2008, David et al., 2014). Given the key role of microbial fermentation in the conversion of algal biomass into absorbable short chain fatty acids (SCFA), this trend of increased or distinct bacterial diversity in herbivores is likely to also hold true for fish. A meta-analysis of the bacterial communities associated with fish suggested that herbivorous species have a core microbiome consisting of members from the order Clostridiales, Bacteroidales and Verrucomicrobiales, all of which are known in other gut systems to be important for the digestion of plant-based materials (Sullam et al., 2012). Sullam et al (2012) further highlight the resemblance between the composition of the gut microbiota of herbivorous fishes with that of 
65 represent the first vertebrate hosts for many of the common gut bacterial taxa (Sullam et al., 66 2012, Wong \& Rawls, 2012).

67

68 Herbivorous fishes play a key role in healthy tropical coral reef ecosystems by consuming 69 seaweeds that can otherwise outcompete corals (McCook et al., 2001). They can consume nearly $70100 \%$ of daily algal primary production in coral reef ecosystems (Carpenter, 1986) and studies 71 from around the world have shown that the absence of these herbivores can result in profound 72 shifts in ecological communities, e.g. from coral to seaweed dominance (Hughes et al., 2007). 73 Rabbitfish belonging to the genus Siganus are one of the most important consumers of 74 macroalgae in Indo-Pacific coral reefs (Bennett \& Bellwood, 2011, Michael et al., 2013, Gilby et 75 al., in Press). Recently, some range-expanding warm-water rabbitfish have also been implicated 76 in the overgrazing of temperate canopy seaweeds around the world, leading to profound shifts in 77 ecological communities from algal forests to turf-dominated systems (Vergés et al., 2014, 78 Bennett et al., 2015, Vergés et al., 2016). Studies of Siganus stellatus show that this species 79 harbors a gut microbial community typical for marine herbivores (Miyake et al., 2015). However 80 the majority of fish microbiome studies such as this one have been performed on faeces or 81 samples of the entire GI tract (i.e. combined content and wall or combined gut regions), thus 82 making the distinction between transient (i.e. allochthonous) food-associated microbiota and the 83 potentially true autochthonous microbiota a challenge. Furthermore studies on insects (e.g. 84 termite) and mammals (e.g. humans) have shown that distinct section of the gut support different 85 microbial communities that are driven by distinct metabolic processes (Brune and Dietrich, 2015, 86 Jandhyala et al., 2015). To what extent fish guts have a similar compartmentalization is however 87 poorly understood.

89 The aim of the present study was to gather baseline data on the microbial communities 90 associated with the gut of $S$. fuscescens, a tropical/ subtropical seaweed-consumer that is linked 91 to the loss of kelp forests in eastern and western Australia as well as Japan (Yamaguchi, 2010, 92 Bennett et al., 2015, Vergés et al., 2016). We examined microbial communities among 93 individuals and compared communities at different gut locations (i.e. midgut and hindgut) and 94 sites within gut locations (i.e. content and wall). This design allowed us to determine if distinct 95 bacterial taxa are enriched in the different gut locations (i.e. midgut and hindgut), if microbial 
96 communities in the gut content (lumen) differed from those associated directly with the gut wall

97 (mucosa), and how the relationship between communities within the content and wall changes 98 along the gut.

99

100

\section{Methods}

101

102

\section{Sampling and DNA extraction}

103 Siganus fuscescens individuals were collected by spearfishing from One Tree Island $\left(23.5076^{\circ} \mathrm{S}\right.$, $\left.104152.0916^{\circ} \mathrm{E}\right)$, in the Southern Great Barrier Reef $(\mathrm{n}=4)$ with permission from the Great Barrier 105 Reef Marine Park (permit G14/36866.10) and the Queensland Fisheries Department (permit 106 170194) and with full University of New South Wales animal ethics approval (permit 13/29A). 107 Fish were collected from coral and sand dominated habitats in lagoon or shallow outer reef 108 habitats. Fish were transported in ice to the laboratory and dissected within 3 hours to separate 109 the entire gut contents, which were stored at $-80^{\circ} \mathrm{C}$ until further processing. No fish with 110 punctured intestinal tracts were used in the analyses. From each fish, the intestinal tract was 111 further dissected using a sterile scalpel to separate the midgut (immediately after the stomach) 112 and the hindgut (immediately before the anus) sections. For each of these two sections, the gut 113 content was squeezed out and the gut wall was separated out. Gut wall samples were washed 114 twice with sterile artificial seawater (ASW) to remove any remaining gut content. Total DNA 115 was extracted from gut content and gut walls using the PowerSoil ${ }^{\circledR}$ DNA isolation kit (MioBio) 116 according to the manufacturers instructions and thereafter stored at $-20^{\circ} \mathrm{C}$.

\section{$11816 \mathrm{~S} r R N A$ gene sequencing and analysis}

120 The 16S rRNA gene was amplified by PCR from total DNA using the method outlined by 121 Lundberg et al. (2013). Briefly, the variable region V4 was targeted with primers 515F and 806R 122 with the PCR including a peptide nucleic acid (PNA) clamp (5'-GGCTCAACCCTGGACAG-3) 123 that suppresses amplification of plastid DNA. PCR products were pooled and sequenced on a 124 MiSeq platform with $2 \times 250$ bp chemistry at the Ramaciotti Centre for Genomics (UNSW). 125 Paired end sequences were merged into contigs, quality filtered, taxonomically classified and 126 clustered into operational taxonomic units (OTUs) using MOTHUR (Schloss et al., 2009) and 
127 the associated MiSeq pipeline (Kozich et al., 2013), but with minor changes. Briefly, singleton

128 contigs were removed after the pre-clustering step, and were classified using the GreenGenes

129 taxonomic outlines (DeSantis et al., 2006) with 60\% confidence threshold.

130

131 Design and statistical analysis

132 The experimental design we used to examine the bacterial communities in the guts of $S$.

133 fuscescens had three factors including fish individuals ('Fish' - four levels), gut location ('GutLoc'

134 - two levels, migut vs hindgut), site ('Site' - two levels, content vs. wall) and the interaction

135 between gut location and site (GutLoc:Site - 4 levels, midgut:content, midgut:wall,

136 hindgut:content, hindgut:wall). We treated Fish as a fixed blocking factor over a random one,

137 since it has been suggested that random factors should have at least five or more levels for

138 efficient estimation of variance parameters (Bates, 2010, Zuur et al., 2013).

139

140 We compared bacterial communities in terms of alpha and beta diversity, and further looked for 141 differentially abundant taxa within communities. For the diversity measures, we first randomly 142 subsampled (rarefied) each sample to a total of 10000 counts to account for uneven sequencing 143 depth among the samples. We conducted this procedure 500 times and took the average to reduce 144 randomisation effects on our subsampled data. The non-subsampled data was used for detecting 145 differentially abundant taxa, as the modeling methods used here (generalised linear models, 146 GLMs) can take effects of sequencing depth into account (using an offset term).

148 The observed number of OTUs and the Shannon diversity coefficient were used as alpha 149 diversity measures for species richness and diversity, respectively. Linear models were 150 constructed and analysis of variance (ANOVA) was used to test for significance of model terms.

151 The Bray-Curtis dissimilarity was used as a beta-diversity measure after square root 152 transformation of relative abundances. Dissimilarities were visualized using Principle Coordinate 153 Analysis (PCoA), and we further used Canonical Analysis of Principal coordinates (CAP) 154 (Anderson MJ \& Willis TJ, 2003) to visualize dissimilarities constrained on each fixed factor 155 (except Fish) within the experimental design (Gut Location, Gut Site and their interaction) and 156 conditional on the Fish factor (i.e. removal of the effect of Fish before visualizing the other 157 factors). Permutational Multivariate Analysis of Variance (PERMANOVA) was used for 
158 hypothesis testing using 999 permutations of the data at hand. Negative binomial GLMs were 159 constructed to detect differentially abundant taxa, as a strong mean-variance relationship of OTU 160 counts was observed. Models were created by taking into account the total number of counts per 161 sample as an offset in the GLM, with the response variable the expected count of an OTU given 162 the sequencing depth. P values were calculated using 999 bootstraps of residuals (resampling 163 rows of the data). Models and $\mathrm{P}$ values were generated using the R package MVAbund (Wang et 164 al., 2012). OTUs were considered to significantly differ between treatments if the likelihood of 165 the observed test statistics was $<5 \%(\mathrm{P}<0.05)$.

166

\section{Results and Discussion}

168

169 The mottled spinefoot rabbitfish (Siganus fuscescens) is a common herbivorous fish found in 170 Indo-Pacific tropical reefs that plays an important role in the control of algal growth on coral reef 171 systems (Bennett \& Bellwood, 2011, Michael et al., 2013, Gilby et al., in Press) yet to date 172 nothing is known about its gut microbiome. Here we investigated the bacterial community in the 173 gut contents and in direct association with the gut wall from individual adults. Bacterial 174 communities were assessed via 16S rRNA gene amplicon sequencing using the Illumina MiSeq 175 platform. Prior to DNA extraction, a high degree of algal material was observed in the gut 176 samples (data not shown), thus samples were amplified with a PNA-clamp designed to bind to 177 plastid DNA (Lundberg et al., 2013). After quality filtering, multiple sequence alignment and 178 clustering at $97 \%$ identity, a total of 1220 OTUs were detected across all 16 samples.

179 Despite the use of the PNA-clamp, there were 25 OTUs (from a total of 1220) classified as 180 chloroplast and these were removed prior to downstream analysis. Rarefaction curves indicated 181 that each sample was sequenced nearly to saturation (Figure S1a) and good coverage was still 182 achieved when the total sequencing depth of each sample was subsampled to 10000 (Figure $183 \mathrm{~S} 1 \mathrm{~b})$.

184

185 We examined alpha diversity within the gut microbiome of $S$. fuscescens using the number of 186 observed OTUs (species richness, Figure S2) and the Shannon-Weaver index (species diversity, 187 Figure 1). Both metrics showed similar patterns, including variation among individual fish, and 188 greater alpha diversity on the gut wall (mucosa) relative to the gut content (lumen) regardless of 
189 gut location (Table S1a and b). While differences in alpha diversity between wall and gut content 190 was generally smaller in the hindgut than in the midgut, the large within group variance and 191 small sample size resulted in limited statistical support for an interaction (Table S1a and b). 192 Interestingly, an individual fish (individual A) showed extreme variation in species diversity, 193 which was due to a greater evenness in the OTU abundance distribution within the midgut of this 194 fish and which was unlike the trends observed in every other sample (Figure 3).

195

196 Analysis of beta diversity using the Bray-Curtis dissimilarity coefficient revealed community 197 dissimilarities ranged between 88.9 to $22.9 \%$. With the exception of the individual A, there was 198 no discernible pattern in the clustering of samples between fish (Figure 2a). Rather, samples 199 tended to cluster according to the gut location (i.e. midgut or hindgut, $\mathrm{F}_{1,9}=5.67, \mathrm{P}<0.001$, 200 Figure $2 \mathrm{~b}$ ) or gut site (i.e. gut wall or gut content, $\mathrm{F}_{1,9}=2.72, \mathrm{P}=0.011$, Figure $2 \mathrm{c}$ ). While 201 bacterial communities of the hindgut content and wall appeared more similar to each other, and 202 together different from the variable communities in the midgut (Figure 2d), there was not enough 203 statistical power to support this observation given the small sample size $\left(\mathrm{F}_{1,9}=1.49, \mathrm{P}=0.133\right.$, 204 Table S1c). It is important to note that while care was taken to avoid sampling bias it is possible 205 that the high variability between samples may partly be explained by technical variability during 206 sample collection (e.g. slight differences in the removal of content or rinsing of the gut walls) in 207 addition to true biological variation.

208

209 Differences in microbiome composition between gut regions and sites (i.e. mucosa and lumen) 210 have previously been reported for Atlantic salmon (Salmo salar) (Gajardo et al., 2016). However 211 in contrast to our observations with S. fuscescens, overall less variation between individual 212 salmon were reported. This finding may relate to general differences between the gut microbiota 213 of herbivores and omnivores (Ley et al., 2008), but could also reflect the defined diet of captive 214 salmon compared to the natural and likely more variable diet of the S. fuscescens. In line with 215 this hypothesis, we found that the variability among fish could be best explained by differences 216 in the microbial community associated with the midgut content rather than differences between 217 other gut locations or sites (Figure 2). While S. fuscescens is known to consume algae, it feeds 218 on both adult macrophytes and the epilithic algal matrix (Wilson et al., 2003), which contains a 219 nutritionally disparate mix of filamentous algae, organic matter, detritus and sand. Further, 
220 closely related species are also known to be opportunistic omnivores, occasionally feeding on 221 invertebrate animals, such as jellyfish (Bos et al., 2016). Thus the high variation in the 222 communities associated with the midgut contents of individual fish could be explained by 223 variation in the food material consumed by the fish prior to collection. These results further 224 highlight the value of sampling from discrete gut regions when assessing the bacterial 225 community of these animals.

226

227 The observation that bacterial communities can vary within the midgut, but tend to become 228 similar to one another towards the hindgut, suggests that specific gut locations select for specific 229 bacterial groups. To determine which bacterial taxa were represented in each of the gut locations 230 and sites, OTUs were taxonomically classified using the GreenGenes taxonomic outlines 231 (DeSantis et al., 2006). In total 25 phyla were observed, however only six of these were found in 232 relative abundances $>0.5 \%$ (Figure S3). Overall delta-Proteobacteria, Firmicutes and 233 Cyanobacteria made up a large proportion of the gut microbiota, and there was clear variation of 234 these taxa across the different gut locations and sites. For example, the Cyanobacteria were 235 found largely within the midgut content, while the delta-Proteobacteria were largely found in the 236 distal gut regardless of the site sampled.

238 At the individual OTU level, only 23 of the total OTU's observed had a relative abundance $>1 \%$ 239 (Figure 3). The most dominant OTU (OTU00001) belonged to the genus Desulfovibrio (delta240 Proteobacteria), which in some samples constituted as much at $60 \%$ of the relative abundance in 241 the community. This OTU was present in all samples, but had a low abundance in the midgut, 242 especially within the content, and a high abundance in the distal gut (Figure 3 ). In contrast, the 243 second most abundant OTU (OTU00002), assigned to the genus Synechococcus, showed the 244 greatest abundance within the midgut content, but was significantly lower within the distal 245 region (Figure 3). Notably, other closely related OTUs had similar patterns in abundance to 246 OTU00001 and OTU00002 with, for example, members of the family Desulfovibrionaceae 247 being in lower abundance within the midgut content and in greater abundance within the midgut 248 wall and the distal gut (Figure S4). In contrast, members of the family Synechococcaceae were 249 only abundant in the midgut content (Figure S4) and thus are likely to be only transient members 250 of these communities. This suggests that specific bacterial groups abundant within the marine 
251 environment (e.g. Synechococcaceae) are largely lost thereafter, while other groups (e.g.

252 Desulfovibrionaceae) can persist, and flourish, while travelling through the gut.

253

254 Given the qualitative observations above, we then modeled OTU counts to detect the number of

255 individual OTUs that were differentially abundant across individuals and within specific sites

256 and gut locations (Figure 4a-b). We found that 39 OTUs varied in abundance across individual

257 fish (Table S2a), while 77 OTUs showed abundance changes across gut locations irrespective of

258 whether we considered the gut wall or the gut contents (Table S2b). 59 OTUs differed across

259 sites irrespective of gut locations (Table S2c) and 50 OTU had abundances that varied depending

260 on both gut locations and sites (Table S2d). Interestingly, while approximately half of the

261 bacteria alternated in abundance between the midgut and hindgut locations (Figure 4b),

262 differences in abundance between gut wall and wall content were largely a result of the majority

263 of OTUs having greater abundances in the wall relative to the content (Figure 4c). Furthermore,

264 OTUs that varied depending on both gut locations and sites tended to have low abundances in the

265 midgut content, but similar abundances among the other gut locations and sites (Figure 4d).

266

267 OTU's enriched in the gut wall belonged to the order Clostridiales and Bacteroidales, including 268 several taxa commonly found in the GI tract of animals, such as Clostridium species and 269 members of the family Rikenellaceae (Rajilić-Stojanović \& de Vos, 2014, Mao et al., 2015).

270 These bacterial groups are efficient fermenters of plant and algal material and thus their close 271 association with the intestinal mucosa presumably ensures direct uptake of fermentation products 272 (i.e. SCFA) to the host bloodstream (Clements et al., 2014, Rajilić-Stojanović \& de Vos, 2014).

273 OTUs that were in greater abundance in the midgut wall, and either site of the hindgut relative to 274 the midgut content were assigned to the families Desulfarculaceae, Rikenellaceae and 275 Ruminococcaceae. Contrasting patterns were observed for OTU's in the family 276 Rhodobacteraceae and genera Rubritalea (Verrucomicrobiaceae). These bacterial taxa are 277 ubiquitous in the marine environment (Brinkhoff et al., 2008, Hedlund et al., 2015) and their 278 greater abundance in the midgut contents compared to the gut wall and hindgut regions suggests 279 that similar to the cyanobacteria, they may represent ingested allochthonous members of the $S$. 280 fuscescens gut microbiome rather than true gut symbionts. OTUs that increased in abundance 281 between the midgut and hindgut $(>0.5 \%$ change) were assigned to the genera Desulfovibrio, 
282 Akkermansia, Treponema and the family Mogibacteriaceae. In contrast, OTUs assigned to the

283 genera Aquimarina, Acinetobacter, and the families Enterobacteriaceae, Peptostreptococcaceae

284 and Pirellulaceae decreased in abundance.

285

286 Overall the observed OTU abundance patterns are likely to reflect changes in the environmental 287 parameters between the gut locations and sites. The enrichment of strictly anaerobic Clostridium 288 species and sulfate reducing bacteria (SRB), such as Desulfovibrio in the hindgut regions implies 289 an anoxic and sulfate-rich environment, which shows a clear compartmentalization of microbial 290 communities across the gut axis driven by host/environmental factors. The abundance of SRB 291 further implies that fermentation of algal material by, for example, Clostridium spp. is likely to 292 be coupled predominately with sulfate reduction rather than acetogenesis or methanogenesis, in 293 line with studies that demonstrated higher rates for sulfate reduction than methanogenesis in the 294 marine herbivorous fish (Mountfort et al., 2002). The dominance of SRB has also been seen for 295 other marine herbivores (Hong et al., 2011), including Siganus stellatus collected from the Red 296 Sea (Miyake et al., 2015), and may reflect a diet rich in sulfated algal polysaccharides. In that 297 regard hindgut processes in herbivorous fish are distinct to those, for example, of termites, which 298 mostly consume low-sulfate organic material and hence couple fermentation to methanogenesis 299 (Brune and Dietrich, 2015).

300

We further suggest marine sediments inadvertently ingested by Siganus sp., while it is feeding on 302 small, benthic algae, as an additional source of both sulfate and SRB. Thus for an actively 303 grazing fish, such as S. fuscescens, the composition of their gut microbiome and the associated metabolic functions are likely to be heavily determined by both the diet and the microorganisms that they ingest from their environment. Similar processes of environmental acquisition, followed by host enrichment of gut bacteria have been suggested for a number of marine and freshwater fish species (Nayak, 2010, Givens et al., 2015, Parris et al., 2016, Yan et al., 2016, Stephens et al., 2016). Therefore in addition to the influence of diet itself (e.g. nutrient content or trophic level), further investigations on the processes that control gut-microbiome assembly in $S$.

310 fuscescens and other marine fish should assess the relative contribution made by diet-associated 311 microorganisms. 
313 In conclusion, despite variation in gut microbial communities across individual S. fuscescens,

314 specific bacterial OTUs were significantly enriched within different locations and sites of the 315 gut. The microbial community associated with samples taken from midgut content was more 316 variable than the other gut samples and contained taxa (e.g. cyanobacteria) that are likely to be 317 associated with what is being ingested with the food, rather than being true members of the gut 318 microbiome. In contrast, the midgut wall, hindgut wall and the hindgut content all appear to 319 support a less transient microbiome, with a higher abundance of taxa known to play an important 320 role in fermentation e.g. Firmicutes and Bacteroidetes. These observations highlight the need for 321 future studies to take into account not only the spatial distribution of bacterial groups along the 322 gastrointestinal tract, but also the relative contribution of environmentally acquired 323 microorganisms to the gut microbiome of ecologically important marine fish.

324

\section{Acknowledgements}

326 We are thankful to Derrick Cruz for his help collecting the fish samples. Sequencing data has 327 been deposited in the NCBI Short Read Archive (SRA) database 328 (https://www.ncbi.nlm.nih.gov/sra) under accession number PRJNA356981.

\section{References}

Anderson MJ \& Willis TJ (2003) Canonical analysis of principal coordinates: a useful method of constrained ordination for ecology. Ecology 84: 511-525.

\section{Bates DM (2010) lme4: Mixed-effects modeling with R. http://lme4.r-forge.r-project.org/book/}

Bennett S \& Bellwood DR (2011) Latitudinal variation in macroalgal consumption by fishes on the Great Barrier Reef. Mar Ecol Prog Ser 426: 241-252.

Bennett S, Wernberg T, Harvey ES \& Santana-Garcon J (2015) Tropical herbivores provide resilience to a climate-mediated phase shift on temperate reefs. Ecol letters 18: 714-723.

Bos AR, Cruz-Rivera E \& Sanad AM (2016) Herbivorous fishes Siganus rivulatus (Siganidae) and Zebrasoma desjardinii (Acanthuridae) feed on Ctenophora and Scyphozoa in the Red Sea. Mar Biodiversity 47:243-246.

Brinkhoff T, Giebel HA \& Simon M (2008) Diversity, ecology, and genomics of the Roseobacter clade: a short overview. Arch Microbiol 189: 531-539. 
349 Brune A \& Dietrich C (2015) The gut microbiota of termintes: digesting the diversity in the light of ecology and evolution. Annu Rev Microbiol 69: 145-166.

351

352

353

354

355

356

357

358

359

360

361

362

363

364

365

366

367

368

369

370

371

372

373

374

375

376

377

Carpenter RC (1986) Partitioning herbivory and its effects on coral-reef algal communities. Ecol Monograph 56: 345-363.

Clements KD, Angert ER, Montgomery WL \& Choat JH (2014) Intestinal microbiota in fishes: what's known and what's not. Mol Ecol 23: 1891-1898

Collins SM \& Bercik P (2009) The relationship between intestinal microbiota and the central nervous system in normal gastrointestinal function and disease. Gastroenterol 136: 2003-2014.

Colston TJ \& Jackson CR (2016) Microbiome evolution along divergent branches of the vertebrate tree of life: what is known and unknown. Mol Ecol 25: 3776-3800.

David LA, Maurice CF, Carmody RN, et al. (2014) Diet rapidly and reproducibly alters the human gut microbiome. Nature 505: 559-563.

DeSantis T, Hugenholtz P, Larsen N, Rojas M, Brodie E, Keller K, Huber T, Dalevi D, Hu P \& Andersen G (2006) Greengenes, a chimera-checked 16S rRNA gene database and workbench compatible with ARB. Appl Environ Microbiol 72: 5069 - 5072.

Gajardo K, Rodiles A, Kortner TM, Krogdahl Å, Bakke AM, Merrifield DL \& Sørum H (2016) A high-resolution map of the gut microbiota in Atlantic salmon (Salmo salar): A basis for comparative gut microbial research. Sci Rep 6: 30893.

Gilby BL, Tibbetts IR \& Stevens T (in Press) Low functional redundancy and high variability in Sargassum browsing fish populations in a subtropical reef system. Mar Fresh Res.

Givens CE, Ransom B, Bano N \& Hollibaugh JT (2015) Comparison of the gut microbiomes of 12 bony fish and 3 shark species. Mar Ecol Prog Ser 518: 209-223.

380

381

382

383

384

385

386

387

388

389

390

391

392

393 Hedlund BP, Yoon J \& Kasai H (2015) Rubritalea. Bergey's Manual of Systematics of Archaea

394 and Bacteria,. John Wiley \& Sons, Ltd. 
395

396

397

398

399

400

401

402

403

404

405

406

407

408

409

410

411

412

413

414

415

416

417

418

419

420

421

422

423

424

425

426

427

428

429

430

431

432

433

434

435

436

437

438

439

Hong PY, Wheeler E, Cann IK \& Mackie RI (2011) Phylogenetic analysis of the fecal microbial community in herbivorous land and marine iguanas of the Galapagos Islands using $16 \mathrm{~S}$ rRNAbased pyrosequencing. ISME J 5: 1461-1470.

Hughes TP, Rodrigues MJ, Bellwood DR, Ceccarelli D, Hoegh-Guldberg O, McCook L, Moltschaniwskyj N, Pratchett MS, Steneck RS \& Willis B (2007) Phase shifts, herbivory, and the resilience of coral reefs to climate change. Curr Biol 17: 360-365.

Jandhyala SM, Talukdar R, Subramanyam C, Vuyyuru H, Sasikala M \& Reddy DN (2015) Role of the normal gut microbiota. World J Gastroenterol 21: 8787-8803.

Kozich JJ, Westcott SL, Baxter NT, Highlander SK \& Schloss PD (2013) Development of a dual-index sequencing strategy and curation pipeline for analyzing amplicon sequence data on the MiSeq Illumina sequencing platform. Appl Environ Microbiol 79: 5112-5120.

Ley RE, Hamady M, Lozupone C, Turnbaugh, PJ, Ramey RR, Bircher JS, Schlegel ML, Tucker TA, Schrenzel MD, Knight R and Gordon JI (2008) Evolution of mammals and their gut microbes. Science 320: 1647-1651.

Lundberg DS, Yourstone S, Mieczkowski P, Jones CD \& Dangl JL (2013) Practical innovations for high-throughput amplicon sequencing. Nat Meth 10: 999-1002.

Mao S, Zhang M, Liu J \& Zhu W (2015) Characterising the bacterial microbiota across the gastrointestinal tracts of dairy cattle: membership and potential function. Sci Rep 5: 16116.

McCook L, Jompa J \& Diaz-Pulido G (2001) Competition between corals and algae on coral reefs: a review of evidence and mechanisms. Coral Reefs 19: 400-417.

Michael PJ, Hyndes GA, Vanderklift MA \& Vergés A (2013) Identity and behaviour of herbivorous fish influence large-scale spatial patterns of macroalgal herbivory in a coral reef. Mar Ecol Prog Ser 482: 227-240.

Miyake S, Ngugi DK \& Stingl U (2015) Diet strongly influences the gut microbiota of surgeonfishes. Mol Ecol 24: 656-672.

Mountfort DO, Campbell J \& Clements KD (2002) Hindgut fermentation in three species of marine herbivorous fish. Appl Environ Microbiol 68: 1374-1380.

Nayak SK (2010) Role of gastrointestinal microbiota in fish. Aquaculture Research 41: $1553-$ 1573.

Parris DJ, Brooker RM, Morgan MA, Dixson DL \& Stewart FJ (2016) Whole gut microbiome composition of damselfish and cardinalfish before and after reef settlement. PeerJ 4: e2412. 
440 Rajilić-Stojanović M \& de Vos WM (2014) The first 1000 cultured species of the human

441

442

443

444

445

446

447

448

449

450

451

452

453

454

455

456

457

458

459

460

461

462

463

464

465

466

467

468

469

470

471

472

473

474

475

476

477

478

479

480

481

482

483

484

485

gastrointestinal microbiota. FEMS Microbiol Rev 38: 996-1047.

Romero J, Ringø E \& Merrifield D (2014) The gut microbiota of fish. Aquaculture Nutrition: Gut Health, Probiotics and Prebiotics, (Merrifield D \& Ringø E, eds.),. John Wiley \& Sons, Ltd, Chichester, UK.

Schloss P, Westcott S, Ryabin T, Hall J, Hartmann M, Hollister E, Lesniewski R, Oakley B, Parks D \& Robinson C (2009) Introducing mothur: Open-Source, Platform-Independent, Community-Supported Software for Describing and Comparing Microbial Communities. Appl Environ Microbiol 75: 7537 - 7541.

Sullam KE, Essinger SD, Lozupone CA, O'Connor MP, Rosen GL, Knight R, Kilham SS \& Russell JA (2012) Environmental and ecological factors that shape the gut bacterial communities of fish: a meta-analysis. Mol Ecol 21: 3363-3378.

Vergés A, Tomas F, Cebrian E, Ballesteros E, Kizilkaya Z, Dendrinos P, Karamanlidis AA, Spiegel D \& Sala E (2014) Tropical rabbitfish and the deforestation of a warming temperate sea. J Ecol 102: 1518-1527.

Vergés A, Doropoulos C, Malcolm HA, Skye M, Gracia-Pizá M, Marzinelli EM, Campbell AH, Ballesteros E, Hoey AS, Vila-Concejo A, Bozec Y and Steinberg PD (2016) Long-term empirical evidence of ocean warming leading to tropicalization of fish communities, increased herbivory and loss of kelp. Proceed Nat Acad Sci USA 113:13791-13796.

Wang Y, Naumann U, Wright ST \& Warton DI. (2012) mvabund - an R package for modelbased analysis of multivariate abundance data. Methods Ecol Evol 3:471-474

Wilson SK, Bellwood DR, Choat JH, \& Furnas MJ (2003) Detritus in the epilithic algal matrix and its use by coral reef fishes. Oceanography and Marine Biology: An Annual Review 41: 279309.

Wong S \& Rawls JF (2012) Intestinal microbiota composition in fishes is influenced by host ecology and environment. Mol Ecol 21: 3100-3102.

Yamaguchi A (2010) Biological aspects of herbivorous fishes in the coastal areas of western Japan. Bull Fish Res Agen 32: 89-94.

Yan Q, Li J, Yu Y, Wang J, He Z, Van Nostrand JD, Kempher ML, Wu L, Wang Y, Liao L, Li $\mathrm{X}$, Wu S, Ni J, Wang C and Zhou J (2016) Environmental filtering decreases with fish development for the assembly of gut microbiota. Environmental Microbiol 18:4739-4754

Stephens WZ, Burns AR, Stagaman K, Wong S, Rawls JF, Guillemin K \& Bohannan BJ (2016) The composition of the zebrafish intestinal microbial community varies across development. ISME J 10: 644-654. 
486 Zuur AF, Hilbe JM \& Ieno EN (2013) A Beginner's Guide to GLM and GLMM with R: A 487 Frequentist and Bayesian Perspective for Ecologists. Highland Statistics Newburgh.

488

489 


\section{Figure legends}

491

492 Figure 1. Bacterial species diversity (Shannon-Weaver diversity index) within the gut of the 493 mottled spinefoot rabbitfish (Siganus fuscescens) among a) individual fish ( $\mathrm{n}=4)$, b) gut 494 locations $(\mathrm{M}=\mathrm{mid}, \mathrm{H}=$ hind $)$, c) gut sites $(\mathrm{C}=$ content, $\mathrm{W}=$ wall $)$ and $\mathrm{d})$ gut sites within gut 495 locations $(\mathrm{MC}=$ mid-content, $\mathrm{MW}=$ mid-wall, $\mathrm{HC}=$ hind-content, $\mathrm{HW}=$ hind-wall $)$, given a 496 total sampling depth of $1000016 \mathrm{~S}$ rRNA gene counts per sample.

497

498

Figure 2. Ordination of Bray-Curtis dissimilarities between bacterial communities within the gut 499 of the mottled spinefoot rabbitfish (Siganus fuscescens) compared among a) individual fish $(\mathrm{n}=$

500 4) using Principle Coordinate Analysis (PCoA), and among b) gut locations $(\mathrm{M}=\mathrm{mid}, \mathrm{H}=$

501 hind $)$, c) gut sites $(\mathrm{C}=$ content, $\mathrm{W}=$ wall $)$ and $\mathrm{d})$ gut sites within gut locations $(\mathrm{MC}=$ mid502 content, $\mathrm{MW}=$ mid-wall, $\mathrm{HC}=$ hind-content, $\mathrm{HW}=$ hind- all) after conditioning on individual 503 fish using Canonical Analysis of Principal coordinates (CAP). Relative abundances, given a total 504 sampling depth of 10000 16S rRNA gene counts per sample, were square root transformed 505 before calculation of dissimilarities. Variance explained in a) for the horizontal axis $=34 \%$ and 506 vertical axis $=23 \%$.

507

508 Figure 3: Relative abundance of bacterial OTUs in the within the gut of the mottled spinefoot 509 rabbitfish (Siganus fuscescens). Only OTUs with relative abundances $>1 \%$ are shown, and are 510 described by the lowest taxonomic assignment. Horizontal axis labels represent Fish individual 511 (A-D) followed by an underscore and then gut sites within gut locations $(\mathrm{MC}=$ mid-content, $512 \mathrm{MW}=$ mid-wall, $\mathrm{HC}=$ hind-content, $\mathrm{HW}=$ hind-wall). 
514 Figure 4: Differentially abundant OTUs within the gut of the mottled spinefoot rabbitfish

515 (Siganus fuscescens) among a) individual fish $(\mathrm{n}=4), \mathrm{b})$ gut locations $(\mathrm{M}=\mathrm{mid}, \mathrm{H}=\mathrm{hind}), \mathrm{c})$

516 gut sites $(\mathrm{C}=$ content, $\mathrm{W}=$ wall $)$ and $\mathrm{d})$ gut sites within gut locations $(\mathrm{MC}=$ mid-content, $\mathrm{MW}=$

517 mid-wall, $\mathrm{HC}=$ hind-content, $\mathrm{HW}=$ hind-wall). OTU abundances have been $\mathrm{z}$-score transformed 518 and thus show the number of standard deviations an OTUs abundance is from the mean 519 abundance of that OTU.

520

521 Figure S1. Rarefaction curves of microbial communities sampled from the gut of mottled 522 spinefoot rabbitfish (Siganus fuscescens), a) after sequence quality filtering and with differential 523 sequencing depths, and b) equalized sampling depths (10 000 sequences randomly obtained per 524 sample).

525

526 Figure S2. Bacterial species richness (number of OTUs) within the gut of the mottled spinefoot 527 rabbitfish (Siganus fuscescens) compared among a) individual fish $(\mathrm{n}=4)$, b) gut locations $(\mathrm{M}=$ 528 mid, $\mathrm{H}=$ hind $), \mathrm{c})$ gut sites $(\mathrm{C}=$ content, $\mathrm{W}=$ wall $)$ and $\mathrm{d})$ gut sites within gut locations $(\mathrm{MC}=$ 529 mid-content, $\mathrm{MW}=$ mid-wall, $\mathrm{HC}=$ hind-content, $\mathrm{HW}=$ hind-wall), given a total sampling 530 depth of $1000016 \mathrm{~S}$ rRNA gene counts per sample.

531

532 Figure S3. Relative abundance of bacterial phyla associated with the GI tract of the mottled 533 spinefoot rabbitfish (Siganus fuscescens). Only phyla with relative abundances $>1 \%$ are shown, 534 and the phylum proteobacteria has been split into it's component classes. Horizontal axis labels 
535 represent Fish individual (A-D) followed by an underscore and then gut sites within gut locations

$536(\mathrm{MC}=$ mid-content, $\mathrm{MW}=$ mid-wall, $\mathrm{HC}=$ hind-content, $\mathrm{HW}=$ hind-wall $)$.

537

538 Figure S4. Standardised abundances (z-score transformation) of OTUs associated with the 539 taxonomic groups o__Desulfovibrionales and f__Synechococcaceae among gut sites within gut 540 locations $(\mathrm{MC}=$ mid-content, $\mathrm{MW}=$ mid-wall, $\mathrm{HC}=$ hind-content, $\mathrm{HW}=$ hind-wall $)$ within the

541 gut of the mottled spinefoot rabbitfish (Siganus fuscescens). Only OTUs with relative 542 abundances $>0.01 \%$ are shown.

543

544

545 


\section{Figure 1 (on next page)}

Figure 1. Bacterial species diversity (Shannon-Weaver diversity index) within the gut of the mottled spinefoot rabbitfish (Siganus fuscescens)

Bacterial species diversity (Shannon-Weaver diversity index) within the gut of the mottled spinefoot rabbitfish (Siganus fuscescens) among a) individual fish $(n=4)$, b) gut locations (M $=$ mid, $\mathrm{H}=$ hind $), c)$ gut sites $(C=$ content, $W=$ wall $)$ and $d)$ gut sites within gut locations ( $M C=$ mid-content, $M W=$ mid-wall, $\mathrm{HC}=$ hind-content, $\mathrm{HW}=$ hind-wall), given a total sampling depth of $1000016 \mathrm{~S}$ rRNA gene counts per sample. 
PeerJ

a)

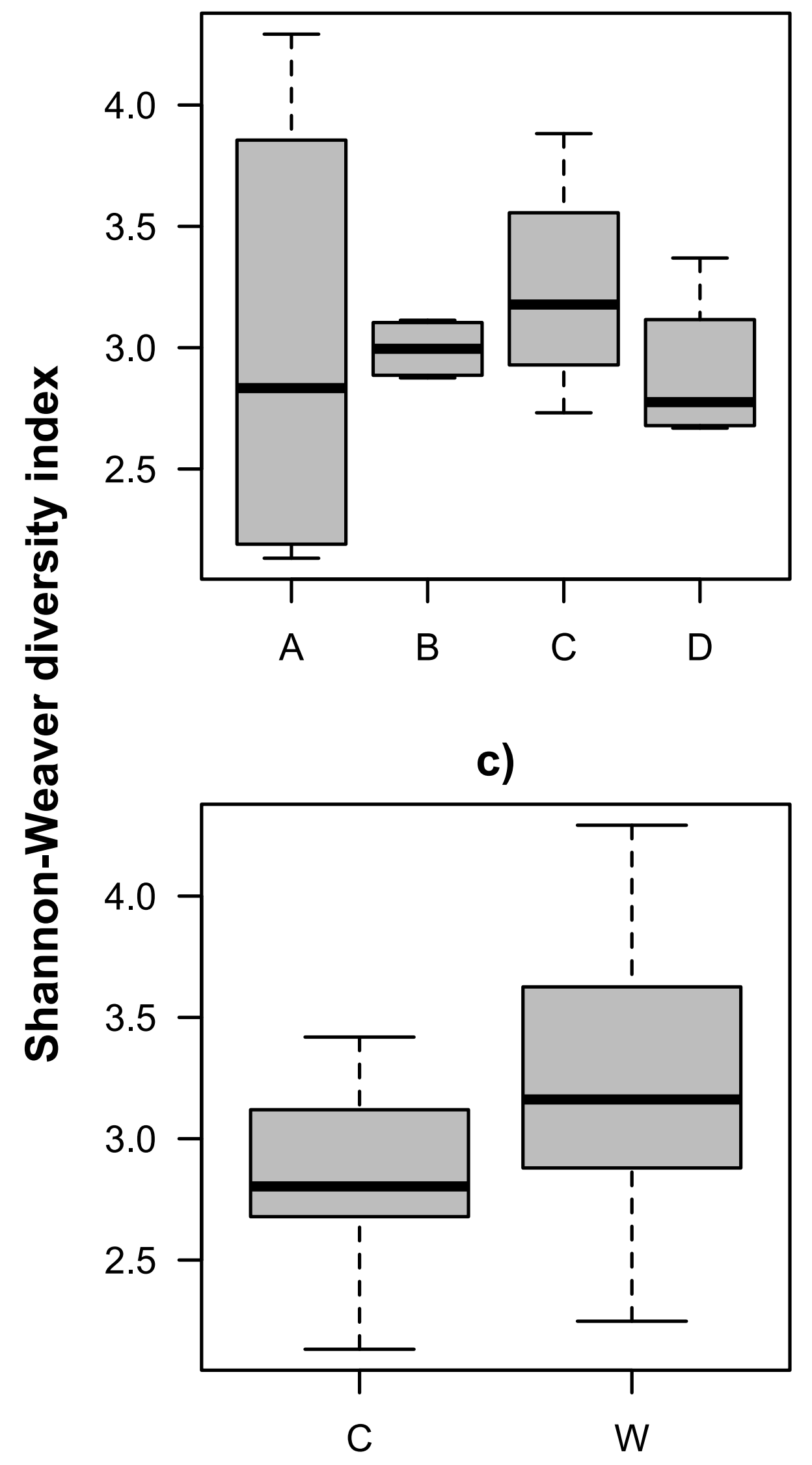

Manuscript to be reviegid

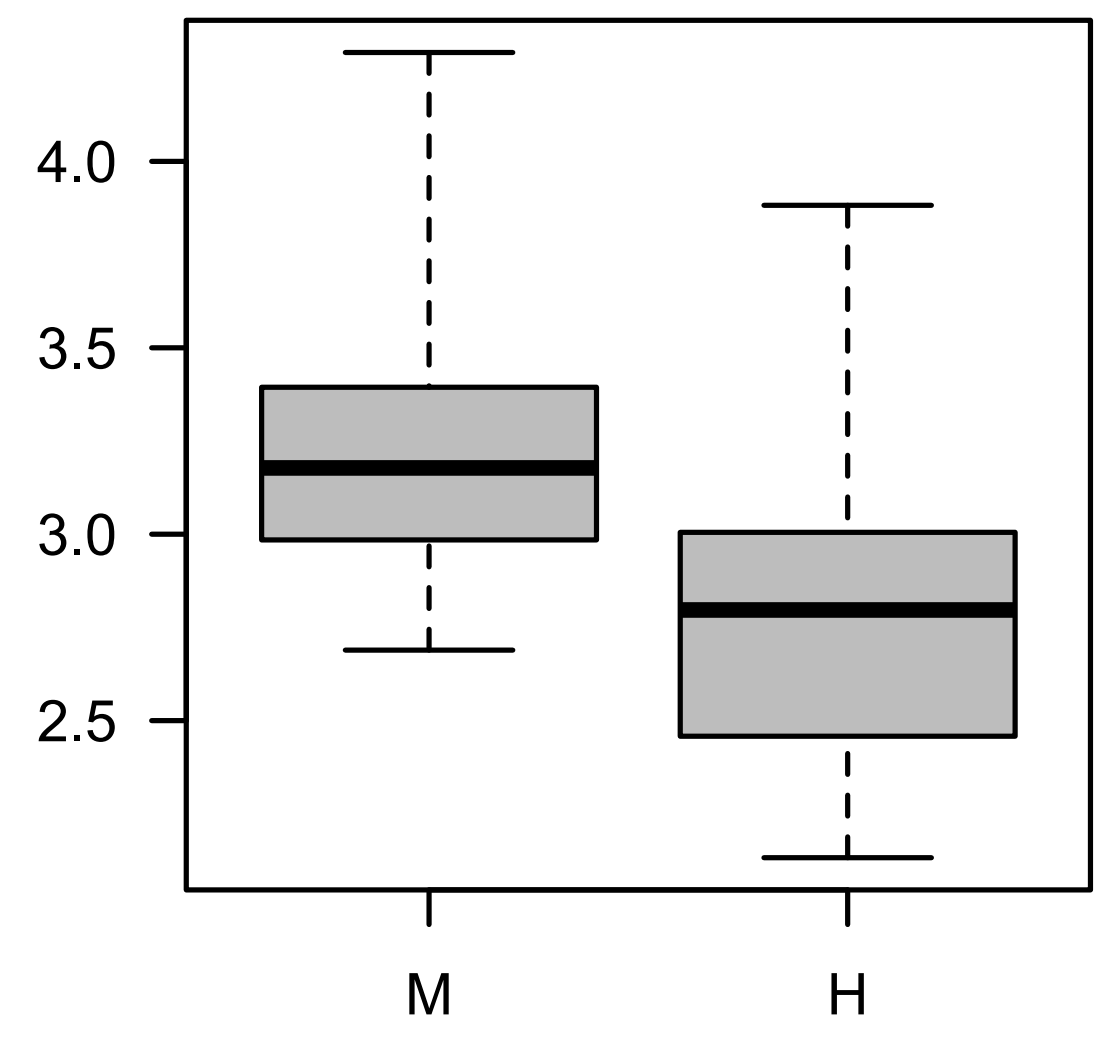

d)

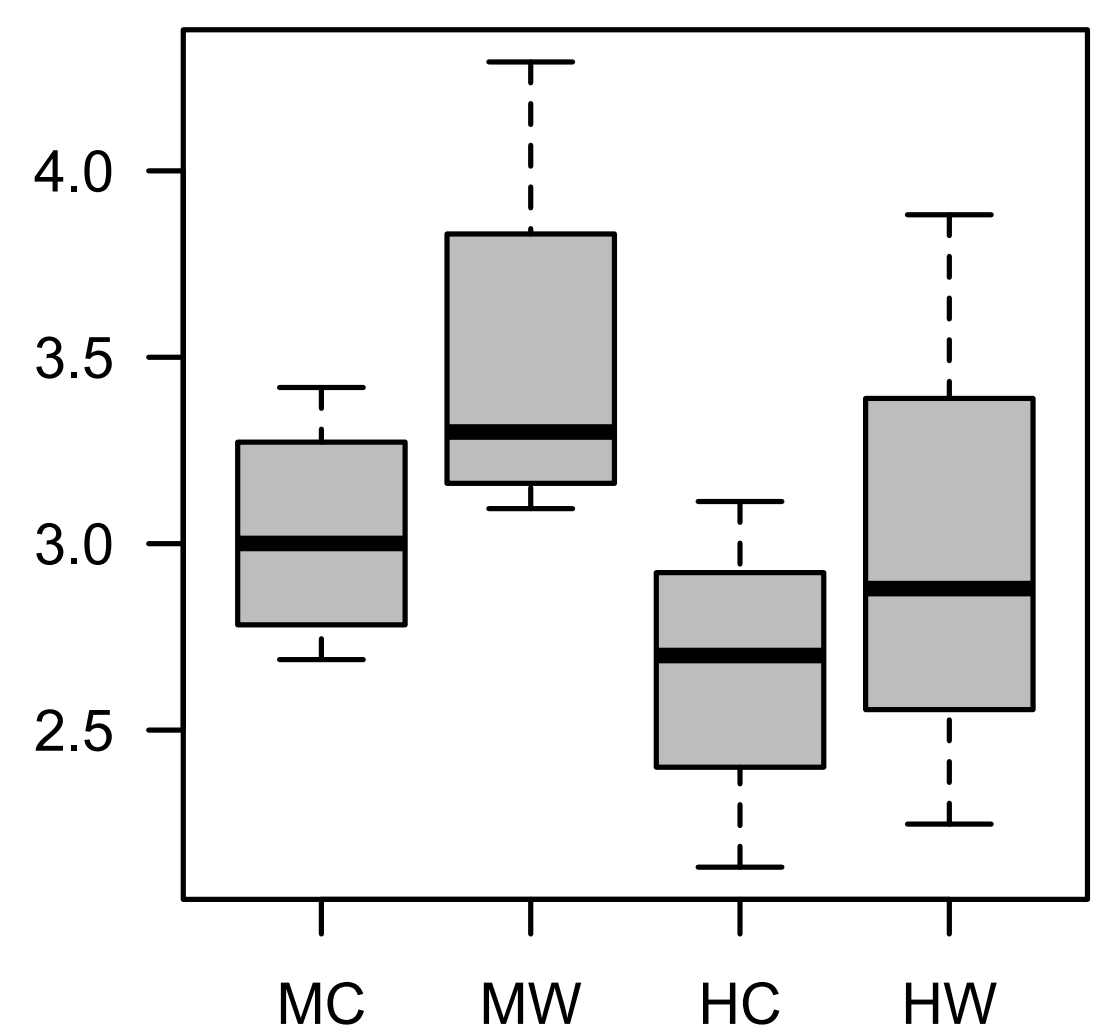




\section{Figure 2 (on next page)}

Figure 2. Principle coordinate analysis (PCoA) ordination of Bray-Curtis dissimilarities between bacterial communities within the gut of the mottled spinefoot rabbitfish (Siganus fuscescens)

Ordination of Bray-Curtis dissimilarities between bacterial communities within the gut of the mottled spinefoot rabbitfish (Siganus fuscescens) compared among a) individual fish $(n=4)$ using Principle Coordinate Analysis ( $P C O A)$, and among b) gut locations ( $M=$ mid, $H=$ hind), c) gut sites ( $C=$ content, $W=$ wall) and $d$ ) gut sites within gut locations ( $M C=$ mid-content, $\mathrm{MW}=$ mid-wall, $\mathrm{HC}=$ hind-content, $\mathrm{HW}=$ hind- all) after conditioning on individual fish using Canonical Analysis of Principal coordinates (CAP). Relative abundances, given a total sampling depth of $1000016 \mathrm{~S}$ rRNA gene counts per sample, were square root transformed before calculation of dissimilarities. Variance explained in a) for the horizontal axis $=34 \%$ and vertical axis $=23 \%$. 
PeerJa)

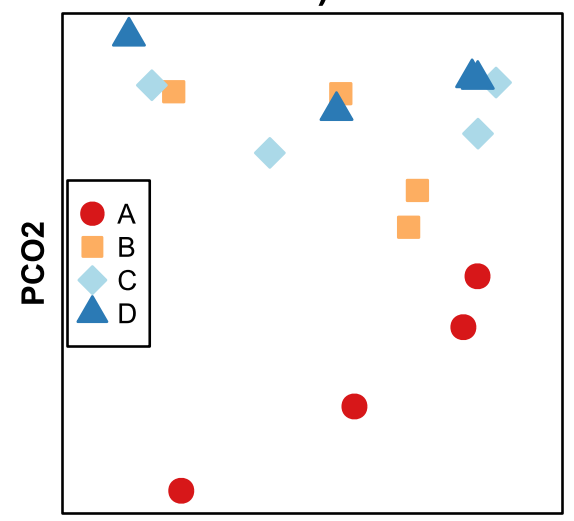

PCO1

c)

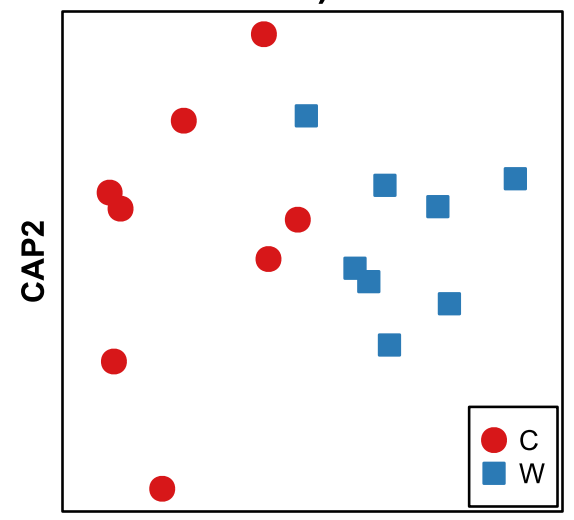

PEARP/PDF | (2017:01:15657:1:1:NEW 14 Mar 2017)
Manuscript to be $\mathbf{b}$ ) /iewed

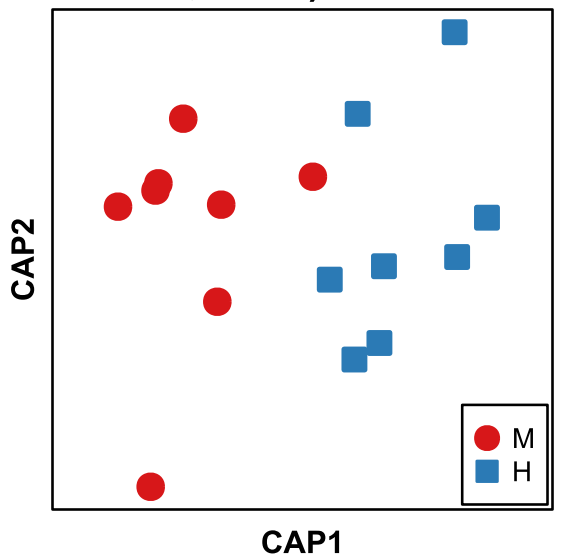

d)

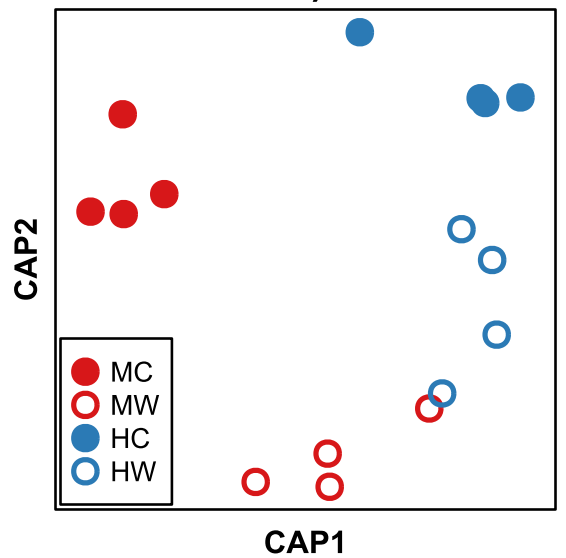




\section{Figure 3 (on next page)}

Figure 3. Relative abundance of bacterial OTUs in the within the gut of the mottled spinefoot rabbitfish (Siganus fuscescens).

Only OTUs with relative abundances $>1 \%$ are shown, and are described by the lowest taxonomic assignment. Horizontal axis labels represent Fish individual (A-D) followed by an underscore and then gut sites within gut locations $(\mathrm{MC}=$ mid-content, $\mathrm{MW}=$ mid-wall, $\mathrm{HC}=$ hind-content, HW = hind-wall). 


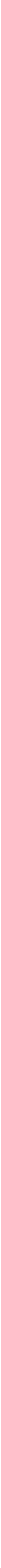




\section{Figure 4(on next page)}

Figure 4. Differentially abundant OTUs within the gut of the mottled spinefoot rabbitfish (Siganus fuscescens)

Differentially abundant OTUs within the gut of the mottled spinefoot rabbitfish (Siganus fuscescens) among a) individual fish $(n=4), b$ ) gut locations ( $M=\operatorname{mid}, H=$ hind), c) gut sites $(C=$ content, $\mathrm{W}=$ wall) and $\mathrm{d}$ ) gut sites within gut locations ( $\mathrm{MC}=$ mid-content, $\mathrm{MW}=$ midwall, $\mathrm{HC}=$ hind-content, $\mathrm{HW}=$ hind-wall). OTU abundances have been $\mathrm{z}$-score transformed and thus show the number of standard deviations an OTUs abundance is from the mean abundance of that OTU. 


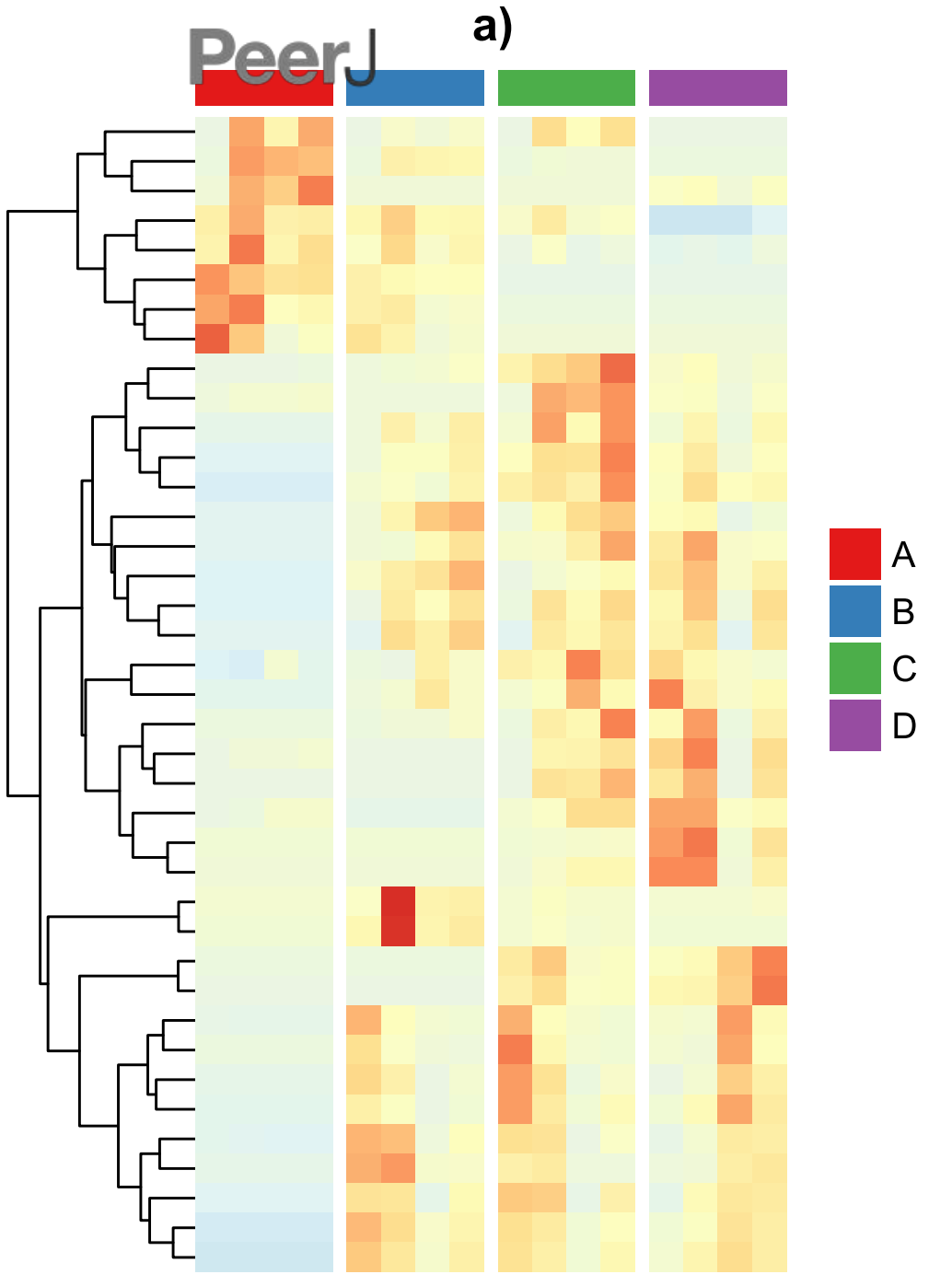

b)

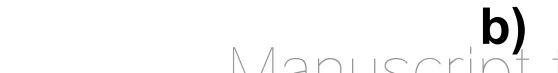

c)
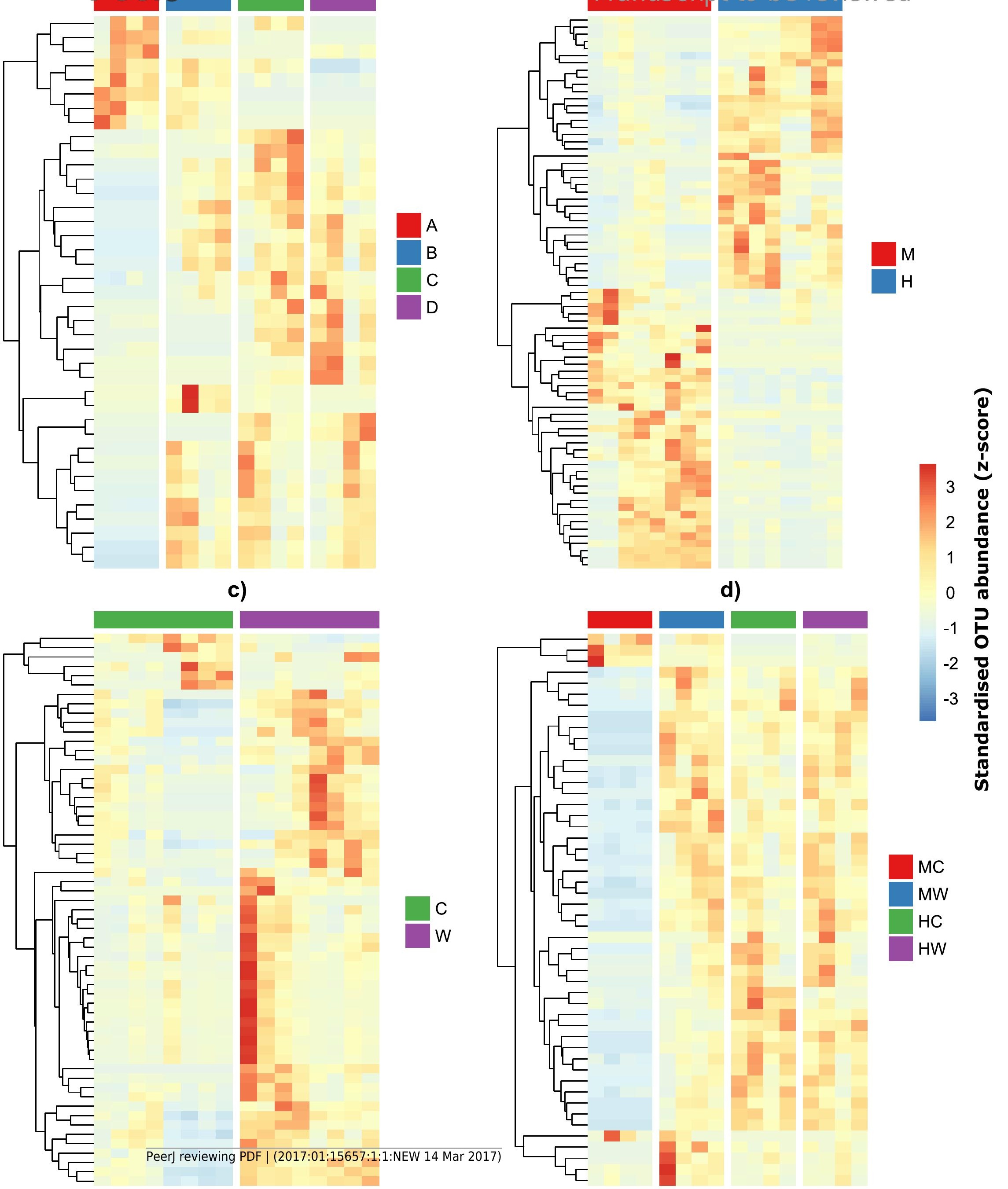